\title{
On generalized convex contractions of type-2 in b-metric and 2-metric spaces
}

\author{
M. S. Khan ${ }^{a}$, Y. Mahendra Singh ${ }^{b}$, Georgeta Maniuc $^{c}$, Mihai Postolache ${ }^{\mathrm{d}, e, *}$ \\ ${ }^{a}$ Department of Mathematics and Statistics, Sultan Qaboos University, P. O. Box 36, PCode 123, Al-Khod, Muscat, Sultanate of Oman. \\ ${ }^{b}$ Department of Humanities and Basic Sciences, Manipur Institute of Technology, Takyelpat-795001, India. \\ ${ }^{c}$ Department of Computer Science, Information Technology, Mathematics and Physics, Petroleum-Gas University of Ploieşti, Bucureşti \\ Bvd., No. 39, 100680 Ploieşti, Romania. \\ ${ }^{d}$ China Medical University, No. 91, Hsueh-Shih Road, Taichung, Taiwan. \\ eDepartment of Mathematics \& Informatics, University "Politehnica" of Bucharest, Splaiul Independentei 313, Bucharest 060042 , \\ Romania.
}

Communicated W. Shatanawi

\begin{abstract}
In this paper, we present the notion of generalized convex contraction mapping of type-2, which includes the generalized convex contraction (resp. generalized convex contraction of order-2) of Miandaragh et al. [M. A. Miandaragh, M. Postolache, S. Rezapour, Fixed Point Theory Appl., 2013 (2013), 8 pages] and the convex contraction mapping of type-2 of Istrățescu [V. I. Istrățescu, I, Libertas Math., 1 (1981), 151-163]. Utilizing this class of mappings, we establish approximate fixed point and fixed point theorems in the setting of b-metric and 2-metric spaces. (c)2017 All rights reserved.
\end{abstract}

Keywords: Approximate fixed point, fixed point, convex contraction, asymptotic regular, $\alpha$-admissible, b-metric and 2-metric spaces.

2010 MSC: 47H10, 54H25.

\section{Introduction and preliminaries}

In 1981, Istrăţescu [17] introduced the class of convex contraction mappings in metric spaces and generalized the well-known Banach's contraction principle [4]. Recently, some works have appeared on the generalization of such classes of mappings in the setting of metric, ordered metric, orthogonal metric and cone metric spaces: Alghamdi et al. [1], Ghorbanian et al. [15], Latif et al. [22], Miandaragh et al. [24], Miculescu and Mihail [26], Ramezani [27], and Sastry et al. [30].

Definition $1.1([3,10])$. Let $X$ be a nonempty set and $s \geqslant 1$ a given real number. A function $d: X \times X \rightarrow$ $[0, \infty)$ is called a $b$-metric on $X$, if for all $x, y, z \in X$, it satisfies:

(i) $d(x, y)=0$, if and only $x=y$;

\footnotetext{
${ }^{*}$ Corresponding author

Email addresses: mohammad@squ.edu.om (M. S. Khan), ymahenmit@rediffmail.com (Y. Mahendra Singh), maniugeorgeta@gmail.com (Georgeta Maniu), mihai@mathem.pub.ro (Mihai Postolache)

doi:10.22436/jnsa.010.06.05
} 
(ii) $d(x, y)=d(y, x)$;

(iii) $d(x, y) \leqslant s[d(x, y)+d(y, z)]$.

The pair $(X, d)$ is called a b-metric space. It is clear that the b-metric space is an extension of the usual metric space. If $s=1$, then it reduces to usual metric space. Therefore, a b-metric space with coefficient $s>1$ need not be a metric space.

The following example of Aydi et al. [2] shows that a b-metric on $X$ need not be a metric on $X$, for more examples one may refer to: Berinde [5], Czerwik [11], Shatanawi et al. [31], Singh and Prasad [32].

Example 1.2. Let $X=\{0,1,2\}$ and $d(2,0)=d(0,2)=m \geqslant 2, d(0,1)=d(1,2)=d(0,1)=d(2,1)=1$ and $d(0,0)=d(1,1)=d(2,2)=0$. Then, $d(x, y) \leqslant \frac{m}{2}[d(x, z)+d(z, y)]$, for all $x, y, z \in X$. If $m>2$, the ordinary triangle inequality does not hold.

For details about the notions of convergence, completeness and Cauchy sequence in the setting of b-metric space, one may refer to Boriceanu et al. [7] and [8].

Definition 1.3 (Gähler [12]). Let $X$ be a nonempty set. If for each $x, y, z \in X$, there exists a real-valued function $d(x, y, z)$ such that:

(i) to each pair of points $x, y$ with $x \neq y$ from $X$, there exists a point $a \in X$ such that $d(x, y, a) \neq 0$;

(ii) $d(x, y, z)=0$, when at least two of the points are equal;

(iii) $d(x, y, z)=d(y, z, x)=d(x, z, y)$;

(iv) $d(x, y, z) \leqslant d(x, y, a)+d(x, a, z)+d(a, y, z)$,

then $d$ is called a 2-metric on $X$ and $(X, d)$ is called a 2-metric space.

It is easily seen that $d$ is non-negative. It has been shown by Gähler [12] that a 2-metric $d$ is a continuous function at any one of its three arguments but it need not be continuous in two arguments. If it is continuous in two arguments, then it is continuous in all three arguments. A 2-metric $d$ which is continuous in all of its arguments will be called continuous.

Example 1.4 (Liu et al. [23]). Let $X=\mathbb{R}^{+} \times \mathbb{R}^{+}$and $d: X \times X \times X \rightarrow[0, \infty)$ a function such that $d(x, y, z)$ is the area of a triangle with vertices $x=\left(x_{1}, x_{2}\right), y=\left(y_{1}, y_{2}\right), z=\left(z_{1}, z_{2}\right)$, for all $x, y, z \in X$. Then $d$ is a 2-metric and $(X, d)$ is a 2-metric space.

Further, for the details about the notions of convergence, Cauchy sequence and completeness in 2metric space, we refer to Gähler [12-14]. Some recent work on fixed point theorems in the setting of 2-metric spaces have appeared in Iśeki [16], Khan [19], Kumar and Poonam [21].

Let $(X, d)$ be a metric space and $T: X \rightarrow X$ be a mapping. With $\varepsilon>0$ be given, then $x_{0} \in X$ is said to be an $\varepsilon$-fixed point of $T$ on $X$, whenever $d\left(x_{0}, T x_{0}\right)<\varepsilon$. Note that every fixed point is an $\varepsilon$-fixed point but the converse need not be true. We denote the set of all $\varepsilon$-fixed points of $T$ for a given $\varepsilon>0$ by $F_{\varepsilon}(T)=\{x \in X \mid d(T x, x)<\varepsilon\}$ and Fix $(T)$, the set of all fixed points of $T$. We say that $T$ has the approximate fixed point property (AFPP) if for all $\varepsilon>0$, there exists an $\varepsilon$-fixed point of T, i.e., for all $\varepsilon, F_{\varepsilon}(T) \neq \emptyset$, or equivalently, $\inf _{x \in X} d(T x, x)=0$, for details, please see: Berinde [6], Kohlenbach and Leuştean [20], Miandaragh et al. [25], Reich and Zaslavski [28], Tijs et al. [33].

Example 1.5. On $X=[0, \infty)$, consider the mapping $T: X \rightarrow X$ given by the formula $T x=x+\frac{1}{2 x+1}$ for all $x \in X$. Setting $0<\varepsilon<\frac{1}{2}$ and taking $x_{0} \in X$ such that $x_{0}>\frac{1-\varepsilon}{2 \varepsilon}$, we obtain,

$$
\mathrm{d}\left(\mathrm{T} x_{0}, \mathrm{x}_{0}\right)=\left|\mathrm{T} x_{0}-\mathrm{x}_{0}\right|=\left|\frac{1}{2 x_{0}+1}\right|<\varepsilon .
$$

This shows that $T$ has an $\varepsilon$-fixed point, so $F_{\varepsilon}(T) \neq \emptyset$. Note that $T$ has no fixed point in $X$. 
Let $\mathrm{T}: X \rightarrow X$ be a self-mapping on a non-empty set $X$ and $\alpha: X \times X \rightarrow[0, \infty)$ be a mapping. We say that $T$ is an $\alpha$-admissible if $x, y \in X, \alpha(x, y) \geqslant 1$ implies that $\alpha(T x, T y) \geqslant 1$; refer to Samet et al. [29]. Also, let $\mathrm{T}: X \rightarrow X$ be a self-mapping on a non-empty set $X$ and $\alpha: X \times X \times X \rightarrow[0, \infty)$ be a mapping, we say that $T$ is an $\alpha$-admissible if $x, y, a \in X, \alpha(x, y, a) \geqslant 1$ implies that $\alpha(T x, T y, a) \geqslant 1$; refer to Kumar and Poonam [21].

We say that $X$ has the property $(H)$ if for each $x, y \in \operatorname{Fix}(T)$, there exists $z \in X$ such that $\alpha(x, z) \geqslant 1$ and $\alpha(y, z) \geqslant 1$; refer to Karapinar [18].

In 2013, Miandaragh et al. [24] introduced the concept of generalized convex contraction (resp. generalized convex contraction of order-2) in the setting of a metric space. A self-mapping $T$ on $X$ is called a generalized convex contraction whenever there exist a mapping $\alpha: X \times X \rightarrow[0, \infty)$ and $\alpha_{1}, \alpha_{2} \in[0,1)$ with $\alpha_{1}+\alpha_{2}<1$ such that

$$
\alpha(x, y) d\left(T^{2} x, T^{2} y\right) \leqslant \alpha_{1} d(x, y)+\alpha_{2} d(T x, T y),
$$

for all $x, y \in X$, where $\alpha$ is the based mapping. Also, a self-mapping $T$ on $X$ is called a generalized convex contraction of order- 2 whenever there exists a mapping $\alpha: X \times X \rightarrow[0, \infty)$ and $\beta_{i}, \gamma_{i} \in[0,1),(i=1,2)$, with $\beta_{1}+\beta_{2}+\gamma_{1}+\gamma_{2}<1$ such that

$$
\alpha(x, y) d\left(T^{2} x, T^{2} y\right) \leqslant \beta_{1} d(x, T x)+\beta_{2} d\left(T x, T^{2} x\right)+\gamma_{1} d(y, T y)+\gamma_{2} d\left(T y, T^{2} y\right),
$$

for all $x, y \in X$, where $\alpha$ is the based mapping.

A self-mapping $T$ on a metric space $(X, d)$ is said to be asymptotically regular [9] at a point $x \in X$ if $\mathrm{d}\left(\mathrm{T}^{\mathrm{n}} x, \mathrm{~T}^{\mathrm{n}+1} \chi\right) \rightarrow 0$ as $\mathrm{n} \rightarrow \infty$.

Lemma $1.6([6])$. Let $(\mathrm{X}, \mathrm{d})$ be a metric space and $\mathrm{T}$ is an asymptotically regular self-mapping on $\mathrm{X}$, i.e., $\mathrm{d}\left(\mathrm{T}^{\mathrm{m}} \chi, \mathrm{T}^{\mathrm{m}+1} \chi\right) \rightarrow 0$, for all $\mathrm{x} \in \mathrm{X}$, then $\mathrm{T}$ has the AFPP.

The following lemma is a direct consequence of Lemma 1.6.

Lemma 1.7 ([22]). Let $(\mathrm{X}, \mathrm{d})$ be a metric space and $\mathrm{T}$ is an asymptotically regular self-mapping at a point $\mathrm{x}_{0} \in \mathrm{X}$, i.e., $\mathrm{d}\left(\mathrm{T}^{\mathrm{m}} \mathrm{x}_{0}, \mathrm{~T}^{\mathrm{m}+1} \mathrm{x}_{0}\right) \rightarrow 0$, then $\mathrm{T}$ has the AFPP.

Recall that a self-mapping $T$ on a 2-metric space $(X, d)$ is said to be asymptotically regular at a point $x \in X$, if $d\left(T^{n} x, T^{n+1} x, a\right) \rightarrow 0$ as $n \rightarrow \infty$, for all $a \in X$.

By analogy to Lemma 1.7, we have the following result.

Lemma 1.8. Let $(\mathrm{X}, \mathrm{d})$ be a 2-metric space and $\mathrm{T}$ be an asymptotically regular self-mapping at a point $\mathrm{x}_{0} \in \mathrm{X}$, i.e., $\mathrm{d}\left(\mathrm{T}^{\mathrm{m}} \mathrm{x}_{0}, \mathrm{~T}^{\mathrm{m}+1} \mathrm{x}_{0}, \mathrm{a}\right) \rightarrow 0$, for all $\mathrm{a} \in \mathrm{X}$, then $\mathrm{T}$ has the AFPP.

Proof. For any integer $n \geqslant 0$, we obtain

$$
\inf _{x \in X} d(x, T x, a) \leqslant d\left(T^{n} x_{0}, T^{n+1} x_{0}, a\right), \quad \forall a \in X .
$$

Since $T$ is asymptotically regular at $x_{0} \in X$, then $d\left(T^{n} x_{0}, T^{n+1} x_{0}, a\right) \rightarrow \infty$ as $n \rightarrow \infty$ which in turn gives $\inf _{x \in X} d(x, T x, a)=0$. That is T has the AFPP.

In this paper, we present the notion of generalized convex contraction mapping of type-2, which includes the generalized convex contraction (resp. generalized convex contraction of order-2) of Miandaragh et al. [24]. Utilizing this class of mapping, we establish approximate fixed point and fixed point theorems in the setting of b-metric and 2-metric spaces.

\section{Main results}

In this section, we extend the notion of convex contraction mapping of type-2 [17] and prove some approximate fixed point and fixed point theorems in the setting of b-metric and 2-metric spaces. 
Definition 2.1. Let $(X, d)$ be a b-metric space with $s \geqslant 1$. A self-mapping $T$ on $X$ is said to be a generalized convex contraction of type-2, if there exist a mapping $\alpha: X \times X \rightarrow[0, \infty)$ and $\alpha_{i}, \beta_{i}, \gamma_{i} \geqslant 0$ with $\sum_{i=1,2}\left(\alpha_{i}+\beta_{i}+\gamma_{i}\right)<1 / s^{2}$ such that

$$
\begin{aligned}
\alpha(x, y) d\left(T^{2} x, T^{2} y\right) \leqslant & \alpha_{1} d(x, y)+\alpha_{2} d(T x, T y)+\beta_{1} d(x, T x)+\beta_{2} d\left(T x, T^{2} x\right) \\
& +\gamma_{1} d(y, T y)+\gamma_{2} d\left(T y, T^{2} y\right)
\end{aligned}
$$

for all $x, y \in X$.

Theorem 2.2. Let $(\mathrm{X}, \mathrm{d})$ be a b-metric space with coefficient $\mathrm{s} \geqslant 1$ and $\mathrm{T}: \mathrm{X} \rightarrow \mathrm{X}$ be a generalized convex contraction of type-2. Suppose that $T$ is $\alpha$-admissible and there exists $x_{0} \in X$ such that $\alpha\left(T x_{0}, x_{0}\right) \geqslant 1$. Then $T$ has an approximate fixed point. Further, $\mathrm{T}$ has a fixed point if $\mathrm{T}$ is continuous and $(\mathrm{X}, \mathrm{d})$ is a complete $\mathrm{b}$-metric space. Moreover, if for all $\mathrm{x}, \mathrm{y} \in \mathrm{Fix}(\mathrm{T})$ we have $\alpha(\mathrm{x}, \mathrm{y}) \geqslant 1$, then $\mathrm{T}$ has a unique fixed point in $\mathrm{X}$.

Proof. Let $x_{0} \in X$ be such that $\alpha\left(T x_{0}, x_{0}\right) \geqslant 1$. Now, we define a sequence $\left\{x_{n}\right\}$ by $x_{n+1}=T^{n+1} x_{0}$, for all $n \geqslant 0$. If $x_{n}=x_{n+1}$, i.e., $T^{n} x_{0}=T\left(T^{n} x_{0}\right)$ for some $n$, then the conclusion of the theorem follows immediately.

Without loss of generality, we assume $x_{n} \neq x_{n+1}$, for all $n \geqslant 0$. Since $T$ is $\alpha$-admissible, $\alpha\left(T x_{0}, x_{0}\right) \geqslant 1$ implies $\alpha\left(T^{2} x_{0}, T x_{0}\right) \geqslant 1$. Therefore, we can obtain inductively that $\alpha\left(T^{n+1} x_{0}, T^{n} x_{0}\right) \geqslant 1$, for all $n \geqslant 0$.

We put $v=\max \left\{d\left(x_{0}, T x_{0}\right), d\left(T x_{0}, T^{2} x_{0}\right)\right\}, \lambda=\sum_{i=1,2}\left(\alpha_{i}+\beta_{i}+\gamma_{i}\right)-\gamma_{2}$ and $\mu=1-\gamma_{2}$. Now, using (2.1), taking $x=x_{0}$ and $y=T x_{0}$, we obtain

$$
\begin{aligned}
d\left(T^{2} x_{0}, T^{3} x_{0}\right) \leqslant & \alpha\left(x_{0}, T x_{0}\right) d\left(T^{2} x_{0}, T^{3} x_{0}\right) \\
\leqslant & \alpha_{1} d\left(x_{0}, T x_{0}\right)+\alpha_{2} d\left(T x_{0}, T^{2} x_{0}\right) \\
& +\beta_{1} d\left(x_{0}, T x_{0}\right)+\beta_{2} d\left(T x_{0}, T^{2} x_{0}\right) \\
& +\gamma_{1} d\left(T x_{0}, T^{2} x_{0}\right)+\gamma_{2} d\left(T^{2} x_{0}, T^{3} x_{0}\right) \\
= & \left(\alpha_{1}+\beta_{1}\right) d\left(x_{0}, T x_{0}\right)+\left(\alpha_{2}+\beta_{2}+\gamma_{1}\right) d\left(T x_{0}, T^{2} x_{0}\right)+\gamma_{2} d\left(T_{0}^{2}, T^{3} x_{0}\right) \\
\leqslant & \left(\alpha_{1}+\alpha_{2}+\beta_{1}+\beta_{2}+\gamma_{1}\right) v+\gamma_{2} d\left(T^{2} x_{0}, T^{3} x_{0}\right) \\
= & \lambda v+\gamma_{2} d\left(T^{2} x_{0}, T^{3} x_{0}\right) .
\end{aligned}
$$

Therefore, we get $\left(1-\gamma_{2}\right) d\left(T^{3} x_{0}, T^{2} x_{0}\right) \leqslant \lambda v$, that is $d\left(T^{2} x_{0}, T^{3} x_{0}\right) \leqslant\left(\frac{\lambda}{\mu}\right) v$, where $\left(\frac{\lambda}{\mu}\right)<1$ as $\sum_{i=1,2}\left(\alpha_{i}+\right.$ $\left.\beta_{i}+\gamma_{i}\right)<1 / s^{2}$.

Again, using (2.1), taking $x=T x_{0}$ and $y=T^{2} x_{0}$, we obtain

$$
\begin{aligned}
d\left(T^{3} x_{0}, T^{4} x_{0}\right) \leqslant & \alpha\left(T x_{0}, T^{2} x_{0}\right) d\left(T^{3} x_{0}, T^{4} x_{0}\right) \\
\leqslant & \alpha_{1} d\left(T x_{0}, T^{2} x_{0}\right)+\alpha_{2} d\left(T^{2} x_{0}, T^{3} x_{0}\right) \\
& +\beta_{1} d\left(T x_{0}, T^{2} x_{0}\right)+\beta_{2} d\left(T^{2} x_{0}, T^{3} x_{0}\right) \\
& +\gamma_{1} d\left(T^{2} x_{0}, T^{3} x_{0}\right)+\gamma_{2} d\left(T^{3} x_{0}, T^{4} x_{0}\right) \\
= & \left(\alpha_{1}+\beta_{1}\right) d\left(T x_{0}, T^{2} x_{0}\right)+\left(\alpha_{2}+\beta_{2}+\gamma_{1}\right) d\left(T^{2} x_{0}, T^{3} x_{0}\right) \\
& +\gamma_{2} d\left(T_{0}^{3}, T^{4} x_{0}\right) \\
\leqslant & \left(\alpha_{1}+\beta_{1}\right) v+\left(\alpha_{2}+\beta_{2}+\gamma_{1}\right)\left(\frac{\lambda}{\mu}\right) v+\gamma_{2} d\left(T_{0}^{3}, T^{4} x_{0}\right) \\
\leqslant & \left(\alpha_{1}+\alpha_{2}+\beta_{1}+\beta_{2}+\gamma_{1}\right) v+\gamma_{2} d\left(T^{3} x_{0}, T^{4} x_{0}\right) .
\end{aligned}
$$

Therefore,

$$
\mathrm{d}\left(\mathrm{T}^{3} x_{0}, \mathrm{~T}^{4} x_{0}\right) \leqslant\left(\frac{\lambda}{\mu}\right) v
$$


and

$$
\begin{aligned}
d\left(T^{4} x_{0}, T^{5} x_{0}\right) \leqslant & \alpha\left(T^{2} x_{0}, T^{3} x_{0}\right) d\left(T^{4} x_{0}, T^{5} x_{0}\right) \\
\leqslant & \alpha_{1} d\left(T^{2} x_{0}, T^{3} x_{0}\right)+\alpha_{2} d\left(T^{3} x_{0}, T^{4} x_{0}\right) \\
& +\beta_{1} d\left(T^{2} x_{0}, T^{3} x_{0}\right)+\beta_{2} d\left(T^{3} x_{0}, T^{4} x_{0}\right) \\
& +\gamma_{1} d\left(T^{3} x_{0}, T^{4} x_{0}\right)+\gamma_{2} d\left(T^{4} x_{0}, T^{5} x_{0}\right) \\
\leqslant & \left(\alpha_{1}+\beta_{1}\right)\left(\frac{\lambda}{\mu}\right) v+\left(\alpha_{2}+\beta_{2}+\gamma_{1}\right)\left(\frac{\lambda}{\mu}\right) v+\gamma_{2} d\left(T^{4} x_{0}, T^{5} x_{0}\right) \\
= & \left(\alpha_{1}+\alpha_{2}+\beta_{1}+\beta_{2}+\gamma_{1}\right)\left(\frac{\lambda}{\mu}\right) v+\gamma_{2} d\left(T^{4} x_{0}, T^{5} x_{0}\right) .
\end{aligned}
$$

It follows

$$
\begin{aligned}
d\left(T^{4} \chi_{0}, T^{5} \chi_{0}\right) & \leqslant \frac{\left(\alpha_{1}+\alpha_{2}+\beta_{1}+\beta_{2}+\gamma_{1}\right)}{\left(1-\lambda_{2}\right)}\left(\frac{\lambda}{\mu}\right) v \\
& =\left(\frac{\lambda}{\mu}\right)^{2} v .
\end{aligned}
$$

Also, we obtain

$$
\begin{aligned}
d\left(T^{5} x_{0}, T^{6} x_{0}\right) \leqslant & \alpha\left(T^{3} x_{0}, T^{4} x_{0}\right) d\left(T^{5} x_{0}, T^{6} x_{0}\right) \\
\leqslant & \alpha_{1} d\left(T^{3} x_{0}, T^{4} x_{0}\right)+\alpha_{2} d\left(T^{4} x_{0}, T^{5} x_{0}\right) \\
& +\beta_{1} d\left(T^{3} x_{0}, T^{4} x_{0}\right)+\beta_{2} d\left(T^{4} x_{0}, T^{5} x_{0}\right) \\
& +\gamma_{1} d\left(T^{4} x_{0}, T^{5} x_{0}\right)+\gamma_{2} d\left(T^{5} x_{0}, T^{6} x_{0}\right) \\
\leqslant & \left(\alpha_{1}+\beta_{1}\right) d\left(T^{3} x_{0}, T^{4} x_{0}\right)+\left(\alpha_{2}+\beta_{2}+\gamma_{1}\right) d\left(T^{4} x_{0}, T^{5} x_{0}\right) \\
& +\gamma_{2} d\left(T^{5} x_{0}, T^{6} x_{0}\right) \\
\leqslant & \left(\alpha_{1}+\beta_{1}\right)\left(\frac{\lambda}{\mu}\right) v+\left(\alpha_{2}+\beta_{2}+\gamma_{1}\right)\left(\frac{\lambda}{\mu}\right) v+\gamma_{2} d\left(T_{0}^{5}, T^{6} x_{0}\right)
\end{aligned}
$$

that is,

$$
\begin{aligned}
d\left(T^{5} x_{0}, T^{6} x_{0}\right) & \leqslant \frac{\left(\alpha_{1}+\alpha_{2}+\beta_{1}+\beta_{2}+\gamma_{1}\right)}{\left(1-\gamma_{2}\right)}\left(\frac{\lambda}{\mu}\right) v \\
& =\left(\frac{\lambda}{\mu}\right)^{2} v .
\end{aligned}
$$

We continue this process, following the similar argument as in [1] and [24], and get $d\left(T^{m} \chi_{0}, T^{m+1} \chi_{0}\right) \leqslant$ $\left(\frac{\lambda}{\mu}\right)^{l} v$, whenever $m=2 l$ or $m=2 l+1$, for $l \geqslant 1$ or $d\left(T^{m} x_{0}, T^{m+1} x_{0}\right) \leqslant\left(\frac{\lambda}{\mu}\right)^{l-1} v$, whenever $m=2 l$ or $m=2 l-1$, for $l \geqslant 2$. Therefore, $d\left(T^{m} x_{0}, T^{m+1} x_{0}\right) \rightarrow 0$ as $m \rightarrow \infty$, i.e., $T$ is asymptotically regular at $x_{0}$. By Lemma 1.7, it follows that $T$ has an approximate fixed point.

Now, suppose that $T$ is continuous and $(X, d)$ is a complete b-metric space. In order to show that $\left\{x_{n}\right\}$ is a Cauchy sequence in $X$, we choose $m, n$ as non-zero positive integers such that $m<n$ with the following cases.

Case (i). For $m=2 l$ with $l, q \geqslant 1$, then

$$
\begin{aligned}
d\left(T^{m} x_{0}, T^{m+q} x_{0}\right)= & d\left(T^{2 l} x_{0}, T^{2 l+q} x_{0}\right) \\
\leqslant & s d\left(T^{2 l} x_{0}, T^{2 l+1} x_{0}\right)+s^{2} d\left(T^{2 l+1} x_{0}, T^{2 l+2} x_{0}\right) \\
& +s^{3} d\left(T^{2 l+2} x_{0}, T^{2 l+3} x_{0}\right)+s^{4} d\left(T^{2 l+3} x_{0}, T^{2 l+4} x_{0}\right)+\cdots
\end{aligned}
$$




$$
\begin{aligned}
& \leqslant s\left(\frac{\lambda}{\mu}\right)^{l} v+s^{2}\left(\frac{\lambda}{\mu}\right)^{l} v+s^{3}\left(\frac{\lambda}{\mu}\right)^{l+1} v+s^{4}\left(\frac{\lambda}{\mu}\right)^{l+1} v+\cdots \\
& \leqslant s\left(\frac{\lambda}{\mu}\right)^{l}\left\{1+s^{2}\left(\frac{\lambda}{\mu}\right)+\cdots\right\} v+s^{2}\left(\frac{\lambda}{\mu}\right)^{l}\left\{1+s^{2}\left(\frac{\lambda}{\mu}\right)+\cdots\right\} v \\
& =\left(s+s^{2}\right)\left(\frac{\lambda}{\mu}\right)^{l}\left\{1+s^{2}\left(\frac{\lambda}{\mu}\right)+\cdots\right\} v \\
& \leqslant\left(s+s^{2}\right)\left(\frac{\lambda}{\mu}\right)^{l} \frac{1}{1-s^{2}\left(\frac{\lambda}{\mu}\right)} v .
\end{aligned}
$$

Similarly, Case (ii). For $m=2 l+1$ with $l, q \geqslant 1$, we obtain

$$
\begin{aligned}
d\left(T^{m} x_{0}, T^{m+q} \chi_{0}\right)= & d\left(T^{2 l+1} x_{0}, T^{2 l+q+1} x_{0}\right) \\
\leqslant & s d\left(T^{2 l+1} x_{0}, T^{2 l+2} \chi_{0}\right)+s^{2} d\left(T^{2 l+2} x_{0}, T^{2 l+3} x_{0}\right) \\
& +s^{3} d\left(T^{2 l+3} x_{0}, T^{2 l+4} x_{0}\right)+s^{4} d\left(T^{2 l+4} x_{0}, T^{2 l+5} x_{0}\right)+\cdots \\
\leqslant & s\left(\frac{\lambda}{\mu}\right)^{l} v+s^{2}\left(\frac{\lambda}{\mu}\right)^{l+1} v+s^{3}\left(\frac{\lambda}{\mu}\right)^{l+1} v+s^{4}\left(\frac{\lambda}{\mu}\right)^{l+2} v+\cdots \\
\leqslant & s\left(\frac{\lambda}{\mu}\right)^{l}\left\{1+s^{2}\left(\frac{\lambda}{\mu}\right)+\cdots\right\} v+s^{2}\left(\frac{\lambda}{\mu}\right)^{l}\left\{1+s^{2}\left(\frac{\lambda}{\mu}\right)+\cdots\right\} v \\
= & \left(s+s^{2}\right)\left(\frac{\lambda}{\mu}\right)^{l}\left\{1+s^{2}\left(\frac{\lambda}{\mu}\right)+\cdots\right\} v \\
\leqslant & \left(s+s^{2}\right)\left(\frac{\lambda}{\mu}\right)^{l} \frac{1}{1-s^{2}\left(\frac{\lambda}{\mu}\right)} v .
\end{aligned}
$$

Taking $l \rightarrow \infty$ in all cases, since $s^{2}\left(\frac{\lambda}{\mu}\right)<1$, we obtain $d\left(T^{m} x_{0}, T^{n} x_{0}\right) \rightarrow 0$. Therefore, $\left\{x_{n}\right\}$ is a Cauchy sequence in $X$. Since, $X$ is complete, there exists a point $z \in X$ such that $x_{n}=T^{n} x_{0} \rightarrow z \in X$ as $n \rightarrow \infty$. By the continuity of $T$, we obtain $z=\lim _{n \rightarrow \infty} T\left(T^{n} x_{0}\right)=T z$. This shows that $z$ is a fixed point of $T$.

Now, we show that $T$ has a unique fixed point in $X$.

In this respect, assume that $z, z^{*} \in \operatorname{Fix}(T)$ such that $z \neq z^{*}$. By the hypothesis $\alpha\left(z, z^{*}\right) \geqslant 1$ and from (2.1) taking $x=z$ and $y=z^{*}$, we obtain

$$
\begin{aligned}
\mathrm{d}\left(z, z^{*}\right)= & d\left(\mathrm{~T}^{2} z, \mathrm{~T}^{2} z^{*}\right) \\
\leqslant & \alpha\left(z, z^{*}\right) \mathrm{d}\left(\mathrm{T}^{2} z, \mathrm{~T}^{2} z^{*}\right) \\
\leqslant & \alpha_{1} \mathrm{~d}\left(z, z^{*}\right)+\alpha_{2} \mathrm{~d}\left(\mathrm{~T} z, \mathrm{~T} z^{*}\right)+\beta_{1} \mathrm{~d}(z, \mathrm{~T} z)+\beta_{2} \mathrm{~d}\left(\mathrm{~T} z, \mathrm{~T}^{2} z\right) \\
& +\gamma_{1} \mathrm{~d}\left(z^{*}, \mathrm{~T} z^{*}\right)+\gamma_{2} \mathrm{~d}\left(\mathrm{~T} z^{*}, \mathrm{~T}^{2} z^{*}\right) \\
\leqslant & \left(\alpha_{1}+\alpha_{2}\right) \mathrm{d}\left(z, z^{*}\right) .
\end{aligned}
$$

It follows that $\left(1-\alpha_{1}-\alpha_{2}\right) d\left(z, z^{*}\right) \leqslant 0$, which in turn gives $d\left(z, z^{*}\right)=0$, a contradiction. Hence $T$ has a unique fixed point in $X$.

Taking $\sum_{i=1,2}\left(\beta_{i}+\gamma_{i}\right)=0$ (resp. $\left.\sum_{i=1,2} \alpha_{i}=0\right)$ with $s=1$ in the inequality (2.1), it reduces to the generalized convex contraction (resp. generalized convex contraction of order-2) of Miandaragh et al. [24].

Corollary 2.3. Let $(\mathrm{X}, \mathrm{d})$ be a b-metric space with coefficient $\mathrm{s} \geqslant 1$ and $\mathrm{T}: \mathrm{X} \rightarrow \mathrm{X}$ be a generalized convex contraction. Suppose that $\mathrm{T}$ is an $\alpha$-admissible and there exists $\mathrm{x}_{0} \in \mathrm{X}$ such that $\alpha\left(\mathrm{T} \mathrm{x}_{0}, \mathrm{x}_{0}\right) \geqslant 1$. Then $\mathrm{T}$ has an approximate fixed point. Further, $\mathrm{T}$ has a fixed point if $\mathrm{T}$ is continuous and $(\mathrm{X}, \mathrm{d})$ is a complete $\mathrm{b}$-metric space. Moreover, if for all $\mathrm{x}, \mathrm{y} \in \mathrm{Fix}(\mathrm{T})$, we have $\alpha(\mathrm{x}, \mathrm{y}) \geqslant 1$, then $\mathrm{T}$ has a unique fixed point in $\mathrm{X}$. 
Proof. Since $\mathrm{T}: \mathrm{X} \rightarrow \mathrm{X}$ is a generalized convex contraction, we obtain

$$
\begin{aligned}
\alpha(x, y) d\left(T^{2} x, T^{2} y\right) \leqslant & \alpha_{1} d(x, y)+\alpha_{2} d(T x, T y) \\
\leqslant & \alpha_{1} d(x, y)+\alpha_{2} d(T x, T y) \\
& +\beta_{1} d(x, T x)+\beta_{2} d\left(T x, T^{2} x\right) \\
& +\gamma_{1} d(y, T y)+\gamma_{2} d\left(T y, T^{2} y\right) .
\end{aligned}
$$

This shows that $\mathrm{T}$ is generalized convex contraction of type-2. Thus, all the conditions of Theorem 2.2 are satisfied, therefore the conclusion follows.

Corollary 2.4. Let $(\mathrm{X}, \mathrm{d})$ be a b-metric space with coefficient $\mathrm{s} \geqslant 1$ and $\mathrm{T}: \mathrm{X} \rightarrow \mathrm{X}$ be a generalized convex contraction of order-2. Suppose that $\mathrm{T}$ is an $\alpha$-admissible and there exists $\mathrm{x}_{0} \in \mathrm{X}$ such that $\alpha\left(\mathrm{T} \mathrm{x}_{0}, \mathrm{x}_{0}\right) \geqslant 1$. Then $\mathrm{T}$ has an approximate fixed point. Further, $\mathrm{T}$ has a fixed point if $\mathrm{T}$ is continuous and $(\mathrm{X}, \mathrm{d})$ is a complete b-metric space. Moreover, if for all $\mathrm{x}, \mathrm{y} \in \mathrm{Fix}(\mathrm{T})$, we have $\alpha(\mathrm{x}, \mathrm{y}) \geqslant 1$, then $\mathrm{T}$ has a unique fixed point in $\mathrm{X}$.

Proof. Since $\mathrm{T}: \mathrm{X} \rightarrow \mathrm{X}$ is generalized convex contraction of order-2, so we have

$$
\begin{aligned}
\alpha(x, y) d\left(T^{2} x, T^{2} y\right) \leqslant & \beta_{1} d(x, T x)+\beta_{2} d\left(T x, T^{2} x\right) \\
& +\gamma_{1} d(y, T y)+\gamma_{2} d\left(T y, T^{2} y\right) \\
\leqslant & \alpha_{1} d(x, y)+\alpha_{2} d(T x, T y) \\
& +\beta_{1} d(x, T x)+\beta_{2} d\left(T x, T^{2} x\right) \\
& +\gamma_{1} d(y, T y)+\gamma_{2} d\left(T y, T^{2} y\right) .
\end{aligned}
$$

It shows that $\mathrm{T}$ is generalized convex contraction of type-2. Thus, all the conditions of Theorem 2.2 are satisfied, therefore the conclusion follows.

Example 2.5. Let $X=[0,1]$ be endowed with $d(x, y)=|x-y|^{2}$. Then, it follows that $d$ is a b-metric on $X$ with coefficient $s=4$. As in [1], we define $T: X \rightarrow X$ by the formula $T x=\frac{x^{2}}{2}+\frac{1}{4}$. Taking $\alpha(x, y)=1$, then $\alpha(T x, T y)=1$, for all $x, y \in X$. Therefore, $T$ is continuous and an $\alpha$-admissible map.

Now, we have

$$
\begin{aligned}
\alpha(x, y) d\left(T^{2} x, T^{2} y\right) & =\left|T^{2} x-T^{2} y\right|^{2} \\
& =\left|\frac{x^{4}+x^{2}+\frac{9}{4}}{8}-\frac{y^{4}+y^{2}+\frac{9}{4}}{8}\right|^{2} \\
& =\frac{1}{64}\left|\left(x^{4}-y^{4}\right)+\left(x^{2}-y^{2}\right)\right|^{2} \\
& \leqslant \frac{1}{16}\left\{\left|x^{4}-y^{4}\right|^{2}+\left|x^{2}-y^{2}\right|^{2}\right\} \\
& \leqslant \frac{1}{8}\left\{\left|x^{2}-y^{2}\right|^{2}+|x-y|^{2}\right\} \\
& \leqslant \frac{1}{2}\left|\frac{x^{2}-y^{2}}{2}\right|^{2}+\frac{1}{8}|x-y|^{2} \\
& \leqslant \frac{1}{2} d(T x, T y)+\frac{1}{8} d(x, y) .
\end{aligned}
$$

It follows that the mapping $T$ is a generalized convex contraction with $\alpha_{1}=\frac{1}{8}$, and $\alpha_{2}=\frac{1}{2}$.

We define a sequence $\left\{x_{n}\right\}$ by $x_{n}=\frac{n}{n+1}-\frac{1}{\sqrt{ } 2}$, then $x_{n} \rightarrow 1-\frac{1}{\sqrt{ } 2}$, as $n \rightarrow \infty$. Therefore, $x_{n+1}=T x_{n}=$ $\left[\frac{1}{2}\left(\frac{n}{n+1}-\frac{1}{\sqrt{ } 2}\right)^{2}+\frac{1}{4}\right] \rightarrow\left(1-\frac{1}{\sqrt{ } 2}\right)$, as $n \rightarrow \infty$. Thus, all the conditions of Corollary 2.3 are satisfied and $x=1-\frac{1}{\sqrt{ } 2}$ is the unique fixed point of $T$ in $X$. 
Now, we introduce the generalized contraction mapping of type-2 in the setting of 2-metric space and prove the following theorem.

Definition 2.6. Let $(X, d)$ be a 2-metric space and $T: X \rightarrow X$ be a self-mapping on $X$. Then $T$ is said to be a generalized convex contraction of type-2, if there exist a mapping $\alpha: X \times X \times X \rightarrow[0, \infty)$ and $\alpha_{i}, \beta_{i}, \gamma_{i} \in[0,1)$ with $\sum_{i=1,2}\left(\alpha_{i}+\beta_{i}+\gamma_{i}\right)<1$ such that

$$
\begin{aligned}
\alpha(x, y, a) d\left(T^{2} x, T^{2} y, a\right) \leqslant & \alpha_{1} d(x, y, a)+\alpha_{2} d(T x, T y, a) \\
& +\beta_{1} d(x, T x, a)+\beta_{2} d\left(T x, T^{2} x, a\right) \\
& +\gamma_{1} d(y, T y, a)+\gamma_{2} d\left(T y, T^{2} y, a\right)
\end{aligned}
$$

for all $x, y, a \in X$.

Theorem 2.7. Let $(\mathrm{X}, \mathrm{d})$ be a 2-metric space and $\mathrm{T}: \mathrm{X} \rightarrow \mathrm{X}$ be a generalized convex contraction of type-2. Suppose that $\mathrm{T}$ is $\alpha$-admissible and there exists $\mathrm{x}_{0} \in \mathrm{X}$ such that $\alpha\left(\mathrm{T} \mathrm{x}_{0}, \mathrm{x}_{0}, \mathrm{a}\right) \geqslant 1$ for all $\mathrm{a} \in \mathrm{X}$. Then, $\mathrm{T}$ has an approximate fixed point. Further, $\mathrm{T}$ has a fixed point if $\mathrm{T}$ is continuous and $(\mathrm{X}, \mathrm{d})$ is a complete 2-metric space. Moreover, if $x, y \in \operatorname{Fix}(T)$, we have $\alpha(x, y, a) \geqslant 1$ for all $a \in X$, then $T$ has a unique fixed point in $X$.

Proof. Let $x_{0} \in X$ be such that $\alpha\left(T x_{0}, x_{0}, a\right) \geqslant 1$, for all $a \in X$. As in Theorem 2.2, we define a sequence $\left\{x_{n}\right\}$ by $x_{n+1}=T^{n+1} x_{0}$, for all $n \geqslant 0$. If $x_{n}=x_{n+1}$, i.e., $T^{n} x_{0}=T\left(T^{n} x_{0}\right)$ for some $n$, then the conclusion follows immediately.

Assume that $x_{n} \neq x_{n+1}$, for all $n \geqslant 0$. Since $T$ is $\alpha$-admissible, $\alpha\left(T x_{0}, x_{0}, a\right) \geqslant 1$, for all $a \in X$ implies that $\alpha\left(T^{2} x_{0}, T x_{0}, a\right) \geqslant 1$. Therefore, we obtain inductively that $\alpha\left(T^{n+1} x_{0}, T^{n} x_{0}, a\right) \geqslant 1$, for all $n \geqslant 0$.

Denote $v=\max \left\{d\left(T x_{0}, T^{2} x_{0}, a\right), d\left(x_{0}, T x_{0}, a\right)\right\}$, for all $a \in X, \lambda=\sum_{i=1,2}\left(\alpha_{i}+\beta_{i}+\gamma_{i}\right)-\gamma_{2}$ and $\mu=$ $1-\gamma_{2}$.

Now, from (2.2) taking with $x=x_{0}$ and $y=T x_{0}$, we obtain

$$
\begin{aligned}
d\left(T^{2} x_{0}, T^{3} x_{0}, a\right) \leqslant & \alpha\left(x_{0}, T x_{0}, a\right) d\left(T^{2} x_{0}, T^{3} x_{0}, a\right) \\
\leqslant & \alpha_{1} d\left(x_{0}, T x_{0}, a\right)+\alpha_{2} d\left(T x_{0}, T^{2} x_{0}, a\right) \\
& +\beta_{1} d\left(x_{0}, T x_{0}, a\right)+\beta_{2} d\left(T x_{0}, T^{2} x_{0}, a\right) \\
& +\gamma_{1} d\left(T x_{0}, T^{2} x_{0}, a\right)+\gamma_{2} d\left(T^{2} x_{0}, T^{3} x_{0}, a\right) \\
= & \left(\alpha_{1}+\beta_{1}\right) d\left(x_{0}, T x_{0}, a\right)+\left(\alpha_{2}+\beta_{2}+\gamma_{1}\right) d\left(T x_{0}, T^{2} x_{0}, a\right) \\
& +\gamma_{2} d\left(T_{0}^{2}, T^{3} x_{0}, a\right) \\
\leqslant & \left(\alpha_{1}+\alpha_{2}+\beta_{1}+\beta_{1}+\gamma_{1}\right) v+\gamma_{2} d\left(T^{2} x_{0}, T^{3} x_{0}, a\right) .
\end{aligned}
$$

Therefore, $d\left(T^{3} x_{0}, T^{2} x_{0}, a\right) \leqslant\left(\frac{\lambda}{\mu}\right) v$. Similarly, we obtain

$$
d\left(T^{3} x_{0}, T^{4} x_{0}, a\right) \leqslant\left(\frac{\lambda}{\mu}\right) v
$$

and

$$
d\left(T^{4} x_{0}, T^{5} x_{0}, a\right) \leqslant\left(\frac{\lambda}{\mu}\right)^{2} v
$$

Also,

$$
d\left(T^{5} x_{0}, T^{6} x_{0}, a\right) \leqslant\left(\frac{\lambda}{\mu}\right)^{2} v
$$

By continuing this process, we obtain $d\left(T^{m} \chi_{0}, T^{m+1} \chi_{0}, a\right) \leqslant\left(\frac{\lambda}{\mu}\right)^{l} v$, whenever $m=2 l$ or $m=2 l+1$, for $l \geqslant 1$ or $d\left(T^{m} x_{0}, T^{m+1} x_{0}, a\right) \leqslant\left(\frac{\lambda}{\mu}\right)^{l-1} v$, whenever $m=2 l$ or $m=2 l-1$, for $l \geqslant 2$. Therefore, 
$\mathrm{d}\left(\mathrm{T}^{\mathrm{m}} \chi_{0}, \mathrm{~T}^{\mathrm{m}+1} \chi_{0}, a\right) \rightarrow 0$ as $\mathrm{m} \rightarrow \infty$, i.e., $T$ is asymptotically regular self-mapping at $x_{0} \in X$, for all $a \in X$. By Lemma 1.8, $\mathrm{T}$ has an approximate fixed point.

Now, suppose that $T$ is continuous and $(X, d)$ is a complete 2-metric space.

In order to show that $\left\{x_{n}\right\}$ is a Cauchy sequence in $X$, we choose $m, n$ as non-zero positive integers such that $m<n$ with the following cases.

Case (i). For $m=2 l$ with $l \geqslant 1$, then

$$
d\left(T^{m} x_{0}, T^{m+1} x_{0}, a\right)=d\left(T^{2 l} x_{0}, T^{2 l+1} x_{0}, a\right) \leqslant\left(\frac{\lambda}{\mu}\right)^{l} v
$$

Now, we have

$$
\begin{aligned}
d\left(T^{m} x_{0}, T^{m+2} x_{0}, a\right)= & d\left(T^{2 l} x_{0}, T^{2 l+2} x_{0}, a\right) \\
\leqslant & d\left(T^{2 l} x_{0}, T^{2 l+2} x_{0}, T^{2 l+1} x_{0}\right)+d\left(T^{2 l} x_{0}, T^{2 l+1}, a\right) \\
& +d\left(T^{2 l+1} x_{0}, T^{2 l+2} x_{0}, a\right) .
\end{aligned}
$$

Therefore, we obtain

$$
\begin{aligned}
d\left(T^{m} x_{0}, T^{m+2} x_{0}, a\right) \leqslant & d\left(T^{2 l} x_{0}, T^{2 l+2} x_{0}, T^{2 l+1} x_{0}\right) \\
& +\sum_{r=0}^{r=1} d\left(T^{2 l+r} x_{0}, T^{2 l+r+1} x_{0}, a\right) .
\end{aligned}
$$

Also, from (2.2), we obtain

$$
\begin{aligned}
d\left(T^{2 l} x_{0}, T^{2 l+2} x_{0}, T^{2 l+1} x_{0}\right)= & d\left(T^{2}\left(T^{2 l-1}\right) x_{0}, T^{2}\left(T^{2 l}\right) x_{0}, T^{2 l} x_{0}\right) \\
\leqslant & \alpha\left(T^{2 l-1} x_{0}, T^{2 l} x_{0}, T^{2 l} x_{0}\right) \\
& d\left(T^{2 l+1} x_{0}, T^{2 l+2} x_{0}, T^{2 l} x_{0}\right) \\
\leqslant & \alpha_{1} d\left(T^{2 l-1} x_{0}, T^{2 l} x_{0}, T^{2 l} x_{0}\right) \\
& +\alpha_{2} d\left(T^{2 l} x_{0}, T^{2 l+1} x_{0}, T^{2 l} x_{0}\right) \\
& +\beta_{1} d\left(T^{2 l-1} x_{0}, T^{2 l} x_{0}, T^{2 l} x_{0}\right) \\
& +\beta_{2} d\left(T^{2 l} x_{0}, T^{2 l+1} x_{0}, T^{2 l} x_{0}\right) \\
& +\gamma_{1} d\left(T^{2 l} x_{0}, T^{2 l+1} x_{0}, T^{2 l} x_{0}\right) \\
& +\gamma_{2} d\left(T^{2 l+1} x_{0}, T^{2 l+2} x_{0}, T^{2 l} x_{0}\right) .
\end{aligned}
$$

Therefore, $\left(1-\gamma_{2}\right) d\left(T^{2 l} x_{0}, T^{2 l+2} x_{0}, T^{2 l+1} x_{0}\right) \leqslant 0$, which in turn gives $d\left(T^{2 l} x_{0}, T^{2 l+2} x_{0}, T^{2 l+1} x_{0}\right)=0$. So, inequality (2.3) reduces to

$$
d\left(T^{m} \chi_{0}, T^{m+2} \chi_{0}, a\right) \leqslant \sum_{r=0}^{r=1} d\left(T^{2 l+r} \chi_{0}, T^{2 l+r+1}, a\right) .
$$

Again, we have

$$
\begin{aligned}
d\left(T^{m} x_{0}, T^{m+3} x_{0}, a\right)= & d\left(T^{2 l} x_{0}, T^{2 l+3} x_{0}, a\right) \\
\leqslant & d\left(T^{2 l} x_{0}, T^{2 l+3} x_{0}, T^{2 l+2} x_{0}\right) \\
& +d\left(T^{2 l} x_{0}, T^{2 l+2} x_{0}, a\right)+d\left(T^{2 l+2} x_{0}, T^{2 l+3} x_{0}, a\right) \\
= & d\left(T^{2 l} x_{0}, T^{2 l+2} x_{0}, T^{2 l+3} x_{0}\right) \\
& +d\left(T^{2 l} x_{0}, T^{2 l+2} x_{0}, a\right)+d\left(T^{2 l+2} x_{0}, T^{2 l+3} x_{0}, a\right),
\end{aligned}
$$


which implies that

$$
d\left(T^{m} x_{0}, T^{m+3} x_{0}, a\right) \leqslant d\left(T^{2 l} x_{0}, T^{2 l+2} x_{0}, T^{2 l+3} \chi_{0}\right)+\sum_{r=0}^{r=2} d\left(T^{2 l+r} x_{0}, T^{2 l+r+1} x_{0}, a\right) .
$$

One can show that $d\left(T^{2 l} x_{0}, T^{2 l+2}, T^{2 l+3}\right)=0$. Similarly, we obtain

$$
d\left(T^{m} x_{0}, T^{m+4} \chi_{0}, a\right) \leqslant \sum_{r=0}^{r=3} d\left(T^{2 l+r} \chi_{0}, T^{2 l+r+1} \chi_{0}, a\right) .
$$

By continuing this process, we obtain

$$
\begin{aligned}
d\left(T^{m} x_{0}, T^{m+q} x_{0}, a\right) \leqslant & \sum_{r=0}^{r=q-1} d\left(T^{2 l+r} x_{0}, T^{2 l+r+1} x_{0}, a\right) \\
= & d\left(T^{2 l} x_{0}, T^{2 l+1} x_{0}, a\right)+d\left(T^{2 l+1} x_{0}, T^{2 l+2} x_{0}, a\right) \\
& +d\left(T^{2 l+2} x_{0}, T^{2 l+3} x_{0}, a\right)+\cdots \\
& +d\left(T^{2 l+q-1} x_{0}, T^{2 l+q} x_{0}, a\right) \\
\leqslant & \left(\frac{\lambda}{\mu}\right)^{l} v+\left(\frac{\lambda}{\mu}\right)^{l} v+\left(\frac{\lambda}{\mu}\right)^{l+1} v+\left(\frac{\lambda}{\mu}\right)^{l+1} v+\cdots \\
\leqslant & 2\left(\frac{\lambda}{\mu}\right)^{l} \frac{1}{1-\left(\frac{\lambda}{\mu}\right)} v .
\end{aligned}
$$

Case (ii). For $m=2 l+1$, where $l \geqslant 1$, then

$$
d\left(T^{m} x_{0}, T^{m+1} x_{0}, a\right)=d\left(T^{2 l+1} x_{0}, T^{2 l+3} x_{0}, a\right) \leqslant\left(\frac{\lambda}{\mu}\right)^{l} v .
$$

Similarly as in Case (i), we obtain

$$
d\left(T^{m} x_{0}, T^{m+3} x_{0}, a\right) \leqslant \sum_{r=0}^{r=2} d\left(T^{2 l+r+1} x_{0}, T^{2 l+r+2} \chi_{0}, a\right),
$$

when $d\left(T^{2 l+1} x_{0}, T^{2 l+3} x_{0}, T^{2 l+4} x_{0}\right)=0$. By continuing this process as in Case (i), we obtain

$$
\begin{aligned}
d\left(T^{m} x_{0}, T^{m+q} x_{0}, a\right) & \leqslant \sum_{r=0}^{r=q-1} d\left(T^{2 l+r} x_{0}, T^{2 l+r+1} x_{0}, a\right) \\
& \leqslant 2\left(\frac{\lambda}{\mu}\right)^{l} \frac{1}{1-\left(\frac{\lambda}{\mu}\right)} v
\end{aligned}
$$

Taking $l \rightarrow \infty$ in all cases, $\left(\frac{\lambda}{\mu}\right)<1$, we obtain $d\left(T^{m} x_{0}, T^{n} x_{0}, a\right) \rightarrow 0$. Therefore, $\left\{x_{n}\right\}$ is a Cauchy sequence in $X$. Since $X$ is a complete 2-metric space, then there exists a point $z \in X$ such that $x_{n}=T^{n} x_{0} \rightarrow$ $z \in X$ as $n \rightarrow \infty$. By the continuity of $T$, we obtain $z=\lim _{n \rightarrow \infty} T\left(T^{n} x_{0}\right)=T z$. This shows that $z$ is a fixed point of $\mathrm{T}$.

Now, we show that $T$ has a unique fixed point in $X$. For this purpose, assume that $z, z^{*} \in \operatorname{Fix}(T)$ such that $z \neq z^{*}$. By the hypothesis $\alpha\left(z, z^{*}, u\right) \geqslant 1$, for all $u \in X$ and from (2.2) taking with $x=z$ and $y=z^{*}$, 
we obtain

$$
\begin{aligned}
d\left(z, z^{*}, u\right)= & d\left(T^{2} z, T^{2} z^{*}, u\right) \\
\leqslant & \alpha\left(z, z^{*}, u\right) d\left(T^{2} z, T^{2} z^{*}, u\right) \\
\leqslant & \alpha_{1} d\left(z, z^{*}, u\right)+\alpha_{2} d\left(T z, T z^{*}, u\right)+\beta_{1} d(z, T z, u)+\beta_{2} d\left(T z, T^{2} z, u\right) \\
& +\gamma_{1} d\left(z^{*}, T z^{*}, u\right)+\gamma_{2} d\left(T z^{*}, T^{2} z^{*}, u\right) \\
\leqslant & \left(\alpha_{1}+\alpha_{2}\right) d\left(z, z^{*}, u\right),
\end{aligned}
$$

implying that $\left(1-\alpha_{1}-\alpha_{2}\right) d\left(z, z^{*}, u\right) \leqslant 0$, which in turn gives $d\left(z, z^{*}, u\right)=0$, a contradiction. Therefore, $T$ has a unique fixed point in $X$.

Taking $\sum_{i=1,2} \alpha_{i}<1$, where $\sum_{i=1,2}\left(\beta_{i}+\gamma_{i}\right)=0$, then inequality (2.2) becomes

$$
\alpha(x, y, a) d\left(T^{2} x, T^{2} y, a\right) \leqslant \alpha_{1} d(x, y, a)+\alpha_{2} d(T x, T y, a) .
$$

Again, taking $\sum_{i=1,2}\left(\beta_{i}+\gamma_{i}\right)<1$, where $\sum_{i=1,2} \alpha_{i}=0$, then inequality (2.2) becomes

$$
\begin{aligned}
\alpha(x, y, a) d\left(T^{2} x, T^{2} y, a\right) \leqslant & \beta_{1} d(x, T x, a)+\beta_{2} d\left(T x, T^{2} x, a\right) \\
& +\gamma_{1} d(y, T y, a)+\gamma_{2} d\left(T y, T^{2} y, a\right) .
\end{aligned}
$$

Note that inequality (2.4) (resp. (2.5)) gives a generalized convex contraction (resp. generalized convex contraction of order-2) in the setting of 2-metric space.

Corollary 2.8. Let $(\mathrm{X}, \mathrm{d})$ be a 2-metric space and $\mathrm{T}: \mathrm{X} \rightarrow \mathrm{X}$ be a generalized convex contraction. Suppose that $\mathrm{T}$ is $\alpha$-admissible and there exists $x_{0} \in X$ such that $\alpha\left(T x_{0}, x_{0}, a\right) \geqslant 1$, for all $\alpha \in X$. Then, $T$ has an approximate fixed point. Further, $\mathrm{T}$ has a fixed point if $\mathrm{T}$ is continuous and $(\mathrm{X}, \mathrm{d})$ is a complete 2-metric space. Moreover, if $x, y \in \operatorname{Fix}(T)$, we have $\alpha(x, y, a) \geqslant 1$ for all $a \in X$, then $T$ has a unique fixed point in $X$.

Corollary 2.9. Let $(X, d)$ be a 2-metric space and $\mathrm{T}: \mathrm{X} \rightarrow \mathrm{X}$ be a generalized convex contraction of order-2. Suppose that $\mathrm{T}$ is $\alpha$-admissible and there exists $\mathrm{x}_{0} \in \mathrm{X}$ such that $\alpha\left(\mathrm{T} \mathrm{x}_{0}, \mathrm{x}_{0}, \mathrm{a}\right) \geqslant 1$, for all $\mathrm{x} \in \mathrm{X}$. Then, $\mathrm{T}$ has an approximate fixed point. Further, $\mathrm{T}$ has a fixed point if $\mathrm{T}$ is continuous and $(\mathrm{X}, \mathrm{d})$ is a complete 2-metric space. Moreover, if $x, y \in \operatorname{Fix}(T)$, we have $\alpha(x, y, a) \geqslant 1$ for all $a \in X$, then $T$ has a unique fixed point in $X$.

\section{Conclusion}

We introduced the notion of generalized convex contraction mapping of type-2, which includes the generalized convex contraction (resp. generalized convex contraction of order-2) of Miandaragh et al. [24] and the convex contraction mapping of type-2 of Istrăţescu [17]. Utilizing this class of mappings, we established approximate fixed point and fixed point theorems in the setting of b-metric and 2-metric spaces.

\section{References}

[1] M. A. Alghamdi, S. H. Alnafei, S. Radenović, N. Shahzad, Fixed point theorems for convex contraction mappings on cone metric spaces, Math. Comput. Modelling, 54 (2011), 2020-2026. 1, 2, 2.5

[2] H. Aydi, M. F. Bota, E. Karapınar, S. Mitrović, A fixed point theorem for set-valued quasi-contractions in b-metric spaces, Fixed Point Theory Appl., 2012 (2012), 8 pages. 1

[3] I. A. Bakhtin, The contraction mapping principle in almost metric space, (Russian) Functional analysis, Ulyanovsk. Gos. Ped. Inst., Ulyanovsk, (1989), 26-37. 1.1

[4] S. Banach, Sur les opérations dans les ensembles abstraits et leur application aux équations intégrales, Fund. Math., 3 (1922), 133-181. 1

[5] V. Berinde, Generalized contractions in quasimetric spaces, Seminar on Fixed Point Theory, "Babeş-Bolyai" Univ., Cluj-Napoca, (1993), 3-9. 1 
[6] M. Berinde, Approximate fixed point theorems, Stud. Univ. Babeş-Bolyai Math., 51 (2006), 11-25. 1, 1.6

[7] M. Boriceanu, Fixed point theory for multivalued generalized contraction on a set with two b-metrics, Stud. Univ. BabeşBolyai Math., 54 (2009), 3-14. 1

[8] M. Boriceanu, M. Bota, A. Petruşsel, Multivalued fractals in b-metric spaces, Cent. Eur. J. Math., 8 (2010), 367-377. 1

[9] F. E. Browder, W. V. Petryshyn, The solution by iteration of nonlinear functional equations in Banach spaces, Bull. Amer. Math. Soc., 72 (1966), 571-575. 1

[10] S. Czerwik, Contraction mappings in b-metric spaces, Acta Math. Inform. Univ. Ostraviensis, 1 (1993), 5-11. 1.1

[11] S. Czerwik, Nonlinear set-valued contraction mappings in b-metric spaces, Atti Sem. Mat. Fis. Univ. Modena, 46 (1998), 263-276. 1

[12] S. Gähler, 2-metrische Räume und ihre topologische Struktur, (German) Math. Nachr., 26 (1963), 115-148. 1.3, 1, 1

[13] S. Gähler, Lineare 2-normierte Räume, (German) Math. Nachr., 28 (1964), 1-43.

[14] S. Gähler, Über 2-Banach-Räume, (German) Math. Nachr., 42 (1969), 335-347. 1

[15] V. Ghorbanian, S. Rezapour, N. Shahzad, Some ordered fixed point results and the property (P), Comput. Math. Appl., 63 (2012), 1361-1368. 1

[16] K. Iséki, Fixed point theorem in 2-metric spaces, Math. Sem. Notes Kobe Univ., 3 (1975), 133-136. 1

[17] V. I. Istrățescu, Some fixed point theorems for convex contraction mappings and convex nonexpansive mappings, I, Libertas Math., 1 (1981), 151-163. 1, 2, 3

[18] E. Karapinar, $\alpha-\psi$-Geraghty contraction type mappings and some related fixed point results, Filomat, 28 (2014), 37-48. 1

[19] M. S. Khan, On fixed point theorems in 2-metric space, Publ. Inst. Math. (Beograd) (N.S.), 41 (1980), 107-113. 1

[20] U. Kohlenbach, L. Leuştean, The approximate fixed point property in product spaces, Nonlinear Anal., 66 (2007), 806818. 1

[21] P. Kumar, Poonam, Fixed point for $\alpha-\psi$ contractive mapping in 2-metric spaces, Int. J. Math. Trends Tech., 10 (2014), 34-37. 1, 1

[22] A. Latif, W. Sintunavarat, A. Ninsri, Approximate fixed point theorems for partial generalized convex contraction mappings in $\alpha$-complete metric spaces, Taiwanese J. Math., 19 (2015), 315-333. 1, 1.7

[23] Z.-Q. Liu, F.-R. Zhang, J.-F. Mao, Common fixed points for compatible mappings of type (A), Bull. Malaysian Math. Soc., 22 (1999), 67-86. 1.4

[24] M. A. Miandaragh, M. Postolache, S. Rezapour, Approximate fixed points of generalized convex contractions, Fixed Point Theory Appl., 2013 (2013), 8 pages. 1, 1, 1, 2, 3

[25] M. A. Miandaragh, M. Postolache, S. Rezapour, Some approximate fixed point results for generalized $\alpha$-contractive mappings, Politehn. Univ. Bucharest Sci. Bull. Ser. A Appl. Math. Phys., 75 (2013), 3-10. 1

[26] R. Miculescu, A. Mihail, A generalization of Istrăţescu's fixed point theorem for convex contractions, ArXiv, 2015 (2015), 17 pages. 1

[27] M. Ramezani, Orthogonal metric space and convex contractions, Int. J. Nonlinear Anal. Appl., 6 (2015), 127-132. 1

[28] S. Reich, A. J. Zaslavski, Genericity and porosity in fixed point theory: a survey of recent results, Fixed Point Theory Appl., 2015 (2015), 21 pages. 1

[29] B. Samet, C. Vetro, P. Vetro, Fixed point theorems for $\alpha$ - $\psi$-contractive type mappings, Nonlinear Anal., 75 (2012), 2154-2165. 1

[30] K. P. R. Sastry, C. S. Rao, A. Chandra Sekhar, M. Balaiah, A fixed point theorem for cone convex contractions of order $m \geqslant 2$, Int. J. Math. Sci. Eng. Appl., 6 (2012), 263-271. 1

[31] W. Shatanawi, A. Pitea, R. Lazović, Contraction conditions using comparison functions on b-metric spaces, Fixed Point Theory Appl., 2014 (2014), 11 pages. 1

[32] S. L. Singh, B. Prasad, Some coincidence theorems and stability of iterative procedures, Comput. Math. Appl., 55 (2008), 2512-2520. 1

[33] S. Tijs, A. Torre, R. Brânzei, Approximate fixed point theorems, Miron Nicolescu (1903-1975) and Nicolae Ciorănescu (1903-1957), Libertas Math., 23 (2003), 35-39. 1 